\title{
ARYL TRIMETHYLAMMONIUM TRIFLUOROMETHANESULFONATES AS \\ PRECURSORS TO ARYL [18F]FLUORIDES
}

M.S. Haka, M.R. Kilbourn, and G.L. Watkins

Division of Nuclear Medicine, Department of Internal Medicine, University of Michigan Medical Center, Ann Arbor, MI 48109

The synthesis of high specific activity aryl [18F]fluorides using nucleophilic aromatic substitution is now well established (1). Most syntheses utilize the [18F]fluoride ion displacement of nitro groups or, less often, halogens $(\mathrm{F}, \mathrm{I}, \mathrm{Br})$. Cationic leaving groups (trimethylammonium salts $(2,3,4,5)$, dimethylsulfonium salts (6) ) have seen less application in fluorine-18 labeling despite the high reactivity of cationic groups towards nucleophilic displacement reactions. Previous experiences with aryl trimethylammonium perchlorates (chosen for the non-nucleophilic nature of the perchlorate counterion) showed facile substitution with [ $\left.{ }^{18} \mathrm{~F}\right] f$ luoride ion, but possible decomposition at elevated temperatures and in one case unacceptable chemical purity of the product, $[18 \mathrm{~F}] \mathrm{N}$-methylspiperone (3). Although not identified as $\left[{ }^{19} \mathrm{~F}\right] \mathrm{N}$-methyl spiperone, the impurity nevertheless lowered the effective specific activity of the final product, and better overall results were obtained using the corresponding nitro precursor. In addition, the synthesis of trimethylammonium perchlorates is lengthy, involving first reaction of silver perchlorate with methyl iodide followed by alkylation of the dimethylamino group, and involves use of potentially explosive perchlorate salts $(2,3)$. Use of the intermediate trimethylammonium iodides in nucleophilic fluorinations did not produce yields equivalent to the nitro precursor. We report here the synthesis and application of aryltrimethylammonium trifluoromethanesulfonates, simple to prepare precursors for aromatic radiofluorination which do not appear to share these problems of decomposition and specific activity.

\section{Synthesis of trifluoromethansulfonate salts. Methyl}

trifluoromethanesulfonate is a readily available, highly reactive methylating reagent (7). Reaction of $\mathrm{N}, \mathrm{N}$-dimethylanilines with this reagent $\left(25^{0} \mathrm{C}\right.$, dichloromethane, $2-12 \mathrm{~h}$ ) produces the desired aryltrimethylammonium trifluoromethansulfonates in one step. The products can be isolated in $>80 \%$ yields by simple recrystallization, and the overall synthesis takes less than 2 days. The methylation conditions are compatible with aldehyde, ketone, ester, and nitrile functional groups, but incompatible with free carboxylic acid groups.

\section{Reactions with No-Carrier-Added [18F]Fluoride ion. Reactions of these} aryltrimethylammonium trifluoromethanesulfonates with NCA $\left[{ }^{18}\right.$ F $]$ fluoride ion are summarized in Table 1. [18F]Fluoride ion was produced by cyclotron irradiation of $[18 \mathrm{O}]$ water, and converted to $\mathrm{NCA}$ tetrabutylammonium 
[18 F]fluoride in dimethylsulfoxide solution by published methods (8).

Resolubolized [18F]fluoride ion was added to $1-2 \mathrm{mg}$ of salt and heated at the designated temperatures for 20-25 minutes (unless noted). Product isolation was by $\mathrm{C}_{18}$ SEP-PAK and identification by silica gel TLC. Yields in Table 1 are based on resolubolized $\left[{ }^{18}\right.$ F]fluoride ion and are corrected for decay.<smiles>CN(c1ccc(P)cc1)[C+](C)(F)F</smiles>

Substrate $(R)$

-COPh

$-\mathrm{CN}$

$-\mathrm{CHO}$

$-\mathrm{COMe}$

$-\mathrm{COOEt}$

*10 min reaction time

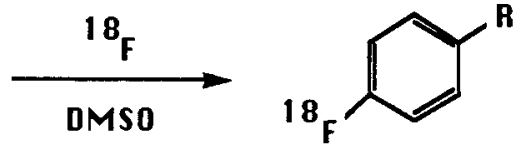

Temperature

$\begin{array}{lll}\frac{140}{78} & 150 & \frac{165}{75} \\ 69 & 65^{*} & 86 \\ & & 74 \\ & & 41\end{array}$

$\frac{120}{80}$
83
70
42
59

59

Syntheses were successful with all of the salts attempted, including the benzaldehyde and acetophenone derivatives which could be considered as highly base-sensitive; previous attempts to prepare p[18F]fluorobenzaldehyde from the corresponding trimethylammonium perchlorate were unsuccessful (2). Yields for the ketones, ester and nitrile derivatives are similar to those reported using the nitro or trimethylammonium perchlorate precursors. There is no apparent change in yields from $100-165{ }^{\circ} \mathrm{C}$, indicating (1) facile displacement of the trimethylammonium group at lower temperatures than possible with the nitro precursors, and (2) no apparent decomposition of the trimethylammonium trifluoromethanesulfonates at higher temperatures $\left(165^{\circ} \mathrm{C}\right)$. In this respect the trifluoromethanesulfonate counterion may be superior to the perchlorate. Acceptable yields can also be obtained with even shorter reaction times than 20 minutes, and in all cases the only fluorine-18 labeld products are the desired aryl [18 F]fluorides.

Isolation of the $18 \mathrm{~F}$-labeled products is straightforward. Both unreacted salt and $\left[{ }^{18}\right.$ F]fluoroaromatic are retained on the $\mathrm{C}_{18}$ SEP-PAK. In most cases, the salts are fairly insoluble in organic solvents, and the salts are not eluted by the organic wash (diethyl ether of dichloromethane). Any traces that do elute can be removed with a short silica gel column. The desired 
[ 18 F]fluoroaromatics are obtained without any UV active impurities (TLC, HPLC), indicating both good separation of the products from starting materials and byproducts, and high specific activity (i.e., no ${ }^{19} \mathrm{~F}$ fluoroaromatics). Exchange of the fluorines in the trifluoromethanesulfonate group with $[18 \mathrm{~F}]$ fluoride thus appears unlikely.

Use of trimethylammonium trifluoromethanesulfonates would thus appear advantageous in the synthesis of high specific activity fluorine-18 labeled radiopharmaceuticals. Preparation of the precursors is simple and efficient, and reaction with $18 \mathrm{~F}$ fluoride ion is high-yielding and can be performed at low temperatures, $\left(100-120{ }^{\circ} \mathrm{C}\right)$. Products can be simply separated from unreacted salt and are obtained in no-carrier-added form, with no apparent dilution of specific activity by adventitious fluoride ion. The application of trifluromethanesulfonate salts to F-18 radiopharmaceutical synthesis is exemplified by the synthesis of F-18 GBR 13119, (1[4-

fluorophenyl)(phenyl)methoxy]-ethyl)-4-(3-phenylpropyl)piperazine), using the benzophenone precursor ( Table $1, \mathrm{R}=\mathrm{COPh}$ ) in an overall 4-step synthesis; the final product can be obtained in $>98 \%$ radiochemical and chemical purity without application of HPLC (use of 4-nitrobenzophenone as precursor requires a final HPLC separation of the corresponding nitro and fluoro compounds) (9).

1) M.S. Berridge and T.J. Tewson, Appl. Radiat Isot 37,685 (1986).

2) G. Angelini, M. Speranza, A.P. Wolf, and C.-Y. Shiue, J. Fluor. Chem. J. of Fluor.

Chem., 27 (1985) 177-191.

3) C.-Y. Shiue, J.S. Fowler, A.P. Wolf, D.W. McPherson, C.D. Arnett, and L. Zecca, J. Nucl. Med. 27: 226-234, 1986.

4) T. Irie, K. Fukushi, O. Inuoe, T. Yamasaki, T. Ido, and T. Nozaki, Int. J. Appl. Isot.

Vol 33, pp 633-636, 1982.

5) T. Irie, K. Fukushi, and T. Ido, Ibid, pp 45-448, 1982.

6) M. Maeda, T. Fukumura, M. Kojima, J. Labeled Cmpd. Radiopharm., 23:1104, 1986.

7) P.J. Stang, and M.R. White, Aldrichimica Acta, Vol. 16, No. 1, 1983.

8) J.W. Brodack, M.R. Kilbourn, M.J. Welch, J.A. Katzenellenhogen, Appl. Radiat. Isot., 37, 217, 1986.

9) M.R. Kilbourn, M.S. Haka, G.K. Mulholland, D.M. Jewett, and D.E. Kuhl, Abstract, Seventh International Symposium on Radiopharmaceutical Chemistry (submitted).

Acknowledgements. This work was supported in part by NIH Grants 2-PO1NS15655 and 1-T32-NS07222-06, and DOE Grant DE-AC02-76EV02031. 